culated $\Gamma_{f} / \Gamma_{n}$ ratios. ${ }^{10}$ Folding the excitation energy distribution into the survival probability, a value of $10^{-32} \mathrm{~cm}^{2}$ is obtained, consistent with the experimental limit mentioned above. Although a similar estimate for $\mathrm{Cf}$ isotopes reproduces the experimental order of magnitude of $\sim 10^{-29} \mathrm{~cm}^{2},{ }^{11}$ the number deduced may only represent an upper limit because of the influence of angular momentum and deformation on the survival probability. It nevertheless appears not to be totally discouraging.

In summary, nuclear reactions of $\mathrm{U}+\mathrm{U}$ are dominated by fission of U-like fragments. The analysis of the surviving part of the binary fragmentation seems to indicate smaller energy dissipation for the same amount of particle diffusion compared to other systems - a feature highly desirable for the synthesis of heavy elements.

The authors want to express their gratitude to the staff of the Unilac for providing the extremely stable $U$ beam. The assistance of the on-line group during the data analysis is gratefully acknowledged.
${ }^{1}$ For the most recent review, see W. U. Schröder and J. R. Huizenga, University of Rochester Report No. UR-NSRL-144, 1977 (unpublished), and to be published.

${ }^{2}$ J. Galin, J. Phys. (Paris), Colloq. 37, C5-83 (1976).

${ }^{3}$ L. G. Moretto and R。 Schmitt, J。 Phys. (Paris), Colloq. 37, C5-109 (1976).

${ }^{4}$ J. R. Huizenga, J. R. Birkelund, W. U. Schröder, K. L. Wolf, and V. E. Viola, Jr., Phys. Rev. Lett. 37 , 885 (1976).

${ }^{5}$ W. Pfeffer, Ph.D. thesis, University of Marburg, 1976 (to be published).

${ }^{6}$ H. Freiesleben, D. v. Harrach, K. D. Hildenbrand, F. Pühlhofer, W. F. W. Schneider, H. J. Specht, and R. Bock, to be published.

${ }^{7}$ R. J. Otto, M. M. Fowler, D. Lee, and G. T. Seaborg, Phys. Rev. Lett. 36, 135 (1976).

${ }^{8}$ J. V. Kratz, A. E. Norris, and G. T. Seaborg, Phys. Rev. Lett. 33, 502 (1974).

${ }^{9}$ W. Norrenberg, Phys. Lett. 52B, 289 (1974), and Z. Phys. A274, 241 (1975), and A276, 84 (1976), and J。 Phys. (Paris), Colloq. 37, C5-141 (1976)。

${ }^{10} \mathrm{~L}$. Moretto, in Proceedings of the Third Symposium on the Physics and Chemistry of Fission, Rochester, New York, 1973 (International Atomic Energy Agency, Vienna, Austria, 1974), Vol. I, p. 329.

${ }^{11}$ J. V. Kratz et al., private communication.

\title{
Formation of Negative Ions in Magnetic Fields
}

\author{
J. Avron, (a) I. Herbst, (b) and B. Simon \\ Joseph Henry Laboratories of Physics, Princeton University, Princeton, New Jersey 08540
}

(Received 9 June 1977)

\begin{abstract}
It is argued that the negative helium ion exists in magnetic fields. Similarly, nontrapping isoelectronic impurities in semiconductors trap with magnetic fields. Crude estimates of binding energies are given.
\end{abstract}

It is well known that a shallow square well will bind in one dimension but not in three. Here we wish to point out an elementary but striking consequence of this fact which affects the physics of atoms in magnetic fields. The new states which we discuss for $\mathrm{He}^{-}$and other ions might make chemistry in strong magnetic fields quite different from conventional chemistry and should be considered in study of stars with large magnetic fields and large concentrations of helium. These states will also have analogs in isoelectronic impurities in solids.

Because of a combination of polarization effects and imperfect shielding, the interaction between a neutral atom and an extra electron is attractive, but as this is the analog of the three-dimensional well, a negative ion will form only if the attraction is sufficiently strong. For example, the com- mon belief is ${ }^{1}$ that noble gases do not have stable negative ions. ${ }^{2,3}$

As distinct from this, constant magnetic fields confine electrons in two dimensions in cyclotron orbits so that the net attractive force will produce a situation analogous to the one-dimensional well, i.e., binding of the electron in the third dimension as well as "tying down" the center of the cyclotron orbit.

In fact, it is not very hard to show ${ }^{4}$ that if $V$ is, for every $\epsilon>0$, the sum of an $L^{2}$ function and a function bounded in absolute value by $\epsilon$, a sufficient condition for the Hamiltonian ${ }^{5}$

$$
H=(2 m)^{-1}(\overrightarrow{\mathrm{p}}-\overrightarrow{\mathrm{A}})^{2}+V(\overrightarrow{\mathrm{r}}) \text {, with } \overrightarrow{\mathrm{A}}=\frac{1}{2} \overrightarrow{\mathrm{B}} \times \overrightarrow{\mathrm{r}},
$$

to have bound states is that $\int V(\overrightarrow{\mathrm{r}}) d^{3} r<0$ or that outside some ball $V(\overrightarrow{\mathrm{r}})<0$. [To see this, use a trial wave function of the form $\varphi_{\lambda}(x, y) f(z)$, for 
$\lambda \geqslant 0$, where $L_{z} \varphi_{\lambda}=\lambda \varphi_{\lambda}$ and $\left\{\left(p_{x}-A_{x}\right)^{2}+\left(p_{y}\right.\right.$ $\left.\left.-A_{y}\right)^{2}\right\} \varphi_{\lambda}=B \varphi_{\lambda}$. This produces an effective potential $W_{\lambda}(z)=\left\langle\varphi_{\lambda}, V(x, y, z) \varphi_{\lambda}\right\rangle$ for a one-dimensional problem which has a bound state if $\int W_{\lambda} d z$ $<0 .{ }^{6}$ Since $\varphi_{\lambda}=($ const $)(x+i y)^{\lambda} e^{-B \rho^{2} / 4}$, where $\rho$ $\equiv\left(x^{2}+y^{2}\right)^{1 / 2}$, if $V<0$ outside some ball, $\int W_{\lambda} d z<0$ for large $\lambda$. Similarly, for suitable positive constants $\beta_{\mu}, \int \sum_{\lambda} \beta_{\lambda} W_{\lambda} d z=\int V d^{3} r$ so that if $\int V d^{3} r<0$, then for some $\lambda, \int W_{\lambda} d z<0$.] From this, it is quite easy to prove rigorously, for example, that $\mathrm{H}^{-}$has an infinity of bound states in a magnetic field $^{4}$ even though it has only one in zero field. ${ }^{7}$ A rigorous proof that $\mathrm{He}^{-}$has a stable bound state turns out to be somewhat involved because of Fermi statistics but can be carried out for small $B .^{4}$ (Unfortunately, a technicality prevents us from proving this for arbitrarily large $B.)^{8}$

As we will see below, the binding energies of these new states are quite small so that we would expect them to be most easily seen experimentally in cases where the only bound states of the system are those produced by the magnetic field acting in concert with the potential. As well as in atoms with negative electron affinities, this situation will occur in some kinds of isoelectronic impurities $^{9}$ in semiconductors which may or may not bind depending on the particular case. ${ }^{9,10}$ Typical nonbinding examples are $\mathrm{Si}: \mathrm{C}$ and $\mathrm{Si}: \mathrm{Ge}$, which will trap in the presence of a magnetic field. The phenomena that we discuss should be distinguished from condensation of impurity states ${ }^{11}$ by the $B$ field. For $B$ small and $\int|V| d^{3} r$ $<\infty$, one can estimate ${ }^{12}$ the binding energy $E(B)$ $\sim \boldsymbol{m}\left[\int V(r) d^{3} \boldsymbol{r}\right]^{2} B^{2}$, so that very roughly $E(B)$ $\sim\left(10^{-16} B^{2}\right)^{\circ} \mathrm{K}$ ( $B$ in gauss). For noble gases $E(B)$ $\sim m \alpha^{2} B^{2}$ with $\alpha$ the polarizability, $m$ the electron mass in a.u.

If $\int|V| d^{3} r=\infty$, then the $B^{2}$ behavior will be replaced by an enhanced behavior. For example, if $V(r) \sim r^{-3}$ for large $r$ (but $V$ not strong enough to bind when $B=0$ ), then $E(B) \sim(B \ln B)^{2}$ for small $B$. It has been suggested ${ }^{10}$ that a deformation potential due to the strain caused by an impurity center may have the general features of the $r^{-3}$ example just discussed. Thus, isoelectronic impurities will have a $B^{2}$ threshold behavior if the mechanism is purely "one-site" as suggested by Hopfield, Dean, and Thomas, ${ }^{13}$ and will have a $(B \ln B)^{2}$ behavior if it is an attractive long-range distortion field.

In any case, for a one-electron Hamiltonian, one has the rigorous bound ${ }^{14,15}$

$$
E(B) \leqslant B / 2 m \text {. }
$$

For $m=1$, the bound is of the order of $\left(10^{-7} B\right)^{\circ} \mathrm{K}$ if $B$ is in gauss. This is small, but not impossible to observe.

It is a pleasure to thank A. K. Ramdas and A. R. Hutson for useful conversations, as well as D. Licciardello for his enthusiasm. This work was supported by National Science Foundation Grants No. MPS 75-22514, No. MPS 74-22844, and No. MPS 75-11864.

\footnotetext{
(a) On leave from Nuclear Research Center-Negev, Beer-Sheva, Israel.

(b) Present address: Department of Mathematics, University of Virginia, Charlottesville, Va. 22903.

${ }^{1}$ There is unfortunately a very limited number of rigorous results on Coulombic binding energies, so that there is no rigorous proof that $\mathrm{He}^{-}$(or even $\mathrm{H}^{--}$!) has no binding. Among the most significant papers on this subject, we mention T. Kato, Trans. Am. Math. Soc.
} 70, 195-211 (1951); M. Zhislin, Trud. Mosc. Math. Soc. 9 , 82-128 (1960); D. Yafeev, Math. Sb. 94, 567 (1974). LMath. USSR Sb. 23, 535 (1974)]; R. N. Hill, Phys. Rev. Lett. 38, 643 (1977).

${ }^{2}$ H. S. W. Massey, Negative Ions (Cambridge Univ. Press, Cambridge, England, '1976), 3rd ed.; H. S. W. Massey and E. H. S. Burhop, Electronic and Ionic Impact Phenomena (Clarendon, Oxford, 1969), Vol. I. We are indebted to Dr. Bahatia for drawing our attention to the fact that the phase shift for electron scattering from He should approach $\pi$ at zero energy because of the closed shell.

${ }^{3}$ H. Hotop and W. C. Lineberger, J. Phys. Chem. Ref. Data 4. 539-576 (1975); R. J. Zollweg, J. Chem. Phys. 50, 4251 (1969).

${ }^{4}$ Various points of rigor will be dealt with in a forthcoming publication.

${ }^{5}$ Below we use atomic units so that $m=1$ is suitable for electrons. The $m$ dependence of various quantities is given for its theoretical interest. In the units we use, $B=1$ is $2.35 \times 10^{9} \mathrm{G}$.

${ }^{6}$ Simon, Ref. 13 .

${ }^{7}$ See Hill, Ref. 1.

${ }^{8} \mathrm{~A}$ crucial step in our proof involves showing that $\mathrm{He}$ in a magnetic field has a nonvanishing quadrupole moment. We are indebted to Professor M. Aizenman for a useful comment on this issue.

${ }^{9}$ For reviews on isoelectronic impurities, see F. Bassani, G. Iandasi, and B. Preziosi, Rep. Prog. Phys. 37, 1039 (1974); D. J. Dean, J. Lumin. 12/13, 83-95 (1976)。Other impurities will always have bound states due to the Coulomb tail.

${ }^{10} \mathrm{~J}$. W. Allen, J. Phys. C 1 , 1136 (1968), and $\underline{4}, 1973$ (1971).

${ }^{11}$ E. H. Putley, J. Phys. Chem. Sol. 21, 241-247 (1961). The binding of the extra electron (hole) is similar to $\mathrm{H}^{-}$, e.g., in Si:P. See C. J. Norton, Phys. Rev. Lett. 37, 164 (1976).

${ }^{12}$ This estimate comes from taking for $\psi(r)$ a function of the form $\alpha(z) \beta(\rho)$, where $z$ is the coordinate parallel 
to the field, and $\rho$ the magnitude of the orthogonal coordinate. We take for $\beta$ the fixed state const. $\times B^{1 / 2}$ $\times e^{-B \rho^{2 / 4}}$ and vary $\alpha$. One then obtains the variational problem for a one-dimensional potential $W(z)$ const. $\times B \int V(\rho, \varphi, z) e^{-B \rho^{2} / 2} \rho d \rho d \varphi$ so that small $B$ corresponds to weak $W$ where the binding energy lsee B. Simon, Ann. Phys. (N.Y.) 97, $279(1976)]$ is $\left[\frac{1}{2} \int W(z) d z\right]^{2}$.

${ }^{13}$ J. J. Hopfield, P. J. Dean, and D. G. Thomas, Phys. Rev. 158, 748-755 (1967).

${ }^{14}$ The inequality (2) comes from the following: The lowest state for which the electron can become unbound has an energy $B / 2 m$ due to the zero-point energy of the Landau oscillator. On the other hand, the energy of the ground state must go up in a magnetic field lsee B. Simon, Phys. Rev. Lett. $\underline{36}, 1083-1084$ (1976)] and we are supposing it to be zero if $B=0$.

${ }^{15}$ If $V(r)=-\alpha /\left(\beta+r^{2}\right)$, with $0 \leqslant 2 m \alpha<1 / 4$ (which implies no bound state if $B=0$ ), one finds by an explicit variational computation that

$$
\lim _{B+0} E(B) / B \geqslant \pi^{3} \alpha^{2} m
$$

which can be made arbitrarily close to $\left(\pi^{3} / 32\right)\left(\frac{1}{2} m\right)$ $=(0.969)\left(\frac{1}{2} m\right)$. This comes close to saturating the bound (2).

\title{
Choice of Gauge in Two-Photon Transitions: $1 s-2 s$ Transition in Atomic Hydrogen
}

\author{
F. Bassani \\ Istituto di Fisica, Università di Roma, Roma, Italy \\ and \\ J. J. Forney and A. Quattropani \\ Laboratoire de Physique Théorique, Ecole Polytechnique Fédérale, Lausanne, Switzerland \\ (Received 25 May 1977)

\begin{abstract}
The two-photon transition rate for hydrogen is evaluated with the $\vec{E} \cdot \vec{x}$ and the $\vec{A} \cdot \vec{p}$ interactions including the continuum. We find that the $\overrightarrow{\mathrm{E}} \cdot \overrightarrow{\mathrm{x}}$ interaction approaches the final result with a small number of intermediate states and consequently is the one to be used in any approximate calculation. Numerical values of the two-photon absorption coefficient as a function of frequency are given and the resonance enhancement is exhibited.
\end{abstract}

The problem of the choice of the gauge in multiphoton transitions has been discussed by a number of author $\mathrm{s}^{1,2}$ in connection with the proper choice of the unperturbed wave functions required to insure gauge invariance. We have shown $n^{2}$ that, at least, in the dipole approximation the two expressions $\vec{E} \cdot \vec{x}$ and $\vec{A} \cdot \vec{p}$ for the photon-electron interaction give the same transition probability to all orders with the same unperturbed wave functions, provided that the complete set of intermediate states is used. In an exact calculation it is thus irrelevant to use one or the other form of the interaction. However, in general only a limited number of intermediate states can be taken into account and the problem of which interaction gives more reliable results in this approximation is essential.

In order to answer this question we have considered a case which can be solved exactly, the two-photon $1 s-2 s$ transition in hydrogen. We present the exact results for the transition rate and absorption coefficient in the two gauges and we compare the convergence over all intermediate states including the continuum. The result of our analysis indicates that the $\overrightarrow{\mathrm{E}} \cdot \overrightarrow{\mathrm{X}}$ interaction is the one to be used in any approximate calculation.

The interaction of radiation with the electron in the two gauges, denoted by $J_{0}$ and $J$, takes the following forms in mks units:

$$
\begin{aligned}
& V^{J_{0}}=-e E_{1} \hat{e}_{1} \cdot \overrightarrow{\mathrm{r}} \cos \omega_{1} t-e E_{2} \hat{e}_{2} \cdot \overrightarrow{\mathrm{r}} \cos \omega_{2} t, \\
& V^{J}=e E_{1} \frac{\hat{e}_{1} \cdot \overrightarrow{\mathrm{p}}}{m \omega_{1}} \sin \omega_{1} t+e E_{2} \frac{\hat{e}_{2} \cdot \overrightarrow{\mathrm{p}}}{m \omega_{2}} \sin \omega_{2} t,
\end{aligned}
$$

where $E_{1}$ and $E_{2}$ are the electric fields of the incident electromagnetic waves traveling in the $y$ direction with polarization vectors $\hat{e}_{1}$ and $\hat{e}_{2}$ and frequencies $\omega_{1}$ and $\omega_{2}$.

The two-photon transition probability per unit time and per atom between the $1 s$ and $2 s$ states reads

$$
\begin{aligned}
& W(1 s-2 s)=\frac{\left|E_{1} E_{2}\right|^{2} e^{4} a_{0}^{4}}{4\left(2 \pi \hbar^{2}\right)^{2} \Re^{3}} \frac{1}{9}\left|D\left[J_{0}\right]\right|^{2} \delta(\Delta \nu), \\
& W(1 s-2 s)=\frac{\left|E_{1} E_{2}\right|^{2} e^{4} a_{0}^{4}}{4\left(2 \pi \hbar^{2}\right)^{2} R^{3}} \frac{1}{9}|D[J]|^{2} \delta(\Delta \nu),
\end{aligned}
$$

in the gauges $J_{0}$ and $J$, respectively. In (2), $a_{0}$ denotes the Bohr radius; $\hat{\mathbb{R}}=3.29 \times 10^{15} \mathrm{sec}^{-1}$, 